\title{
Applications of Electrospun Fibers in Sensors
}

\author{
Caihong Zhu ${ }^{1, a^{*}}$ and Chengwei Wang ${ }^{1, b}$ \\ ${ }^{1}$ Suzhou Vocational University, Suzhou, China \\ azhuzhang_2000@163.com, bch@jssvc.edu.cn \\ ${ }^{*}$ The corresponding author
}

\begin{abstract}
Keywords: Electrospun fiber; Biosensor; Gas sensor; Metalion sensor
\end{abstract}
\begin{abstract}
Electrospun fibers with many advantages such as nano-structure, large specific surface area, high porosity, have been widely applied in the field of sensors. This paper mainly introduces latest development of electrospun fiber applications in biosensors, gas sensors and metal ion sensors. Biosensors are introduced according to the types of sensitive materials (enzyme, metal oxides, other biological materials except enzyme) immobilizated on electrospun fibers. Gas sensors based on electrospun fibers are simply summaried in organic gas sensors and inorganic gas sensors, and some typical examples are introduced. Some novel copper ion sensors based on electrospun fibers are also discussed to example for the electrospun fiber applications in metal ion sensors. In the conclusion part, two aspects are proposed to improve the applications of electrospun fibers in sensors.
\end{abstract}

\section{Introduction}

Electrospinning is one of the effective methods of obtaining nanofibers. Since electrostatic spinning device was prepared for producing superfine fibers by polymer materials in the thirties of twentieth century [1], electrospinning technology has been rapidly developed. In 1971, Baumgarten for the first time obtained electrospun fibers with diameters reaching nano level. In 1979, Walter invented no-nozzle electrospinning device and this potently enchanced the efficiency of producing electrospun fibers. With the rise of nanotechnology in the last ten years, the electrospinning technique has also been considerable developed. Electrospun fibers with diameters between several tens to several hundred nanometers can be obtained and electrospun materials have become more widely including polymers, mixtures, semiconductors, ceramics, etc, that has directly contributed to wide applications of electrospun fibers in scientific research and production practice. Now electrospun fibers have been broadly used in human tissue regeneration scaffold [2], drug release system, various types of battery, catalyst and sensors, etc. A set of conventional electrostatic spinning apparatus is shown in Fig. 1 [18], usually containing high-voltage power supply system, injection system and receiving system. Electrospinning principle is that, with the raise of the electric field caused by the increasing voltage, the charged droplet at the spinneret is stretched gradually and forms a Taylor cone at a equilibrium state when the voltage reaches a certain value. Then the charged liquid drops under the action of electric field force, jets from the Taylor cone and forms a high speed jet. The high speed jet is strongly streached and the solvent volatilizes quickly in the movement, which makes the charge density increases quickly. The high density of charge leads to refining division of the jet, resulting in superfine fibers collected by the receiving system.

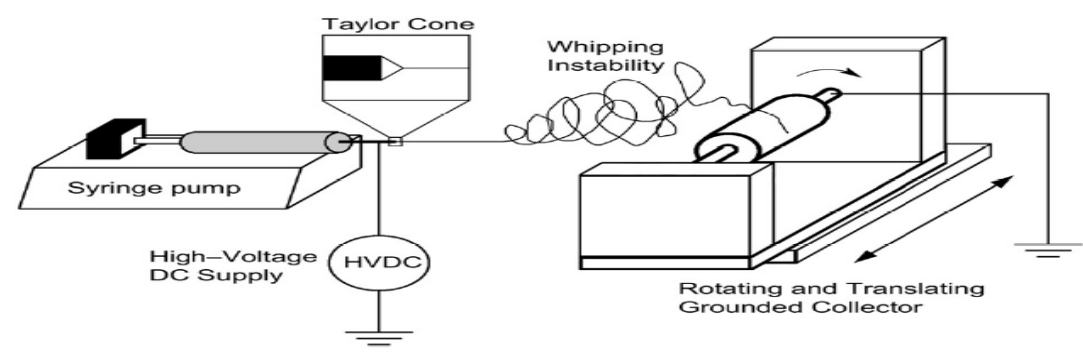

Figure 1. A set of conventional electrostatic spinning apparatus 
As the most important part of information collection, the sensor technology is one of the three pillars (the other two are communication technology and computer technology) of the information industry. A variety of sensor types has appeared, such as sound wave sensor, resistance sensor and optical sensor [3].The materials which can be detected by sensors have also been very widely, including biological molecule, various types of gases, metal ionsand so on. The principle of sensor for detection of substances is showed in Fig. 2. Performance indexes of sensor for detecting substances, such as sensitivity, response time and stability, depend largely on the performance of sensing material carrier. Now semiconductor and carbon graphite materials are widely applied as the sensing material carriers. The electrospun fibers with nanodiameter, large specific surface area, high porosity, etc., become an excellent candidate for the sensitive substance carrier. This paper has mainly introduced the latest research progress of electrospun fiber applications in biological sensors, gas sensors and metal ion sensors.

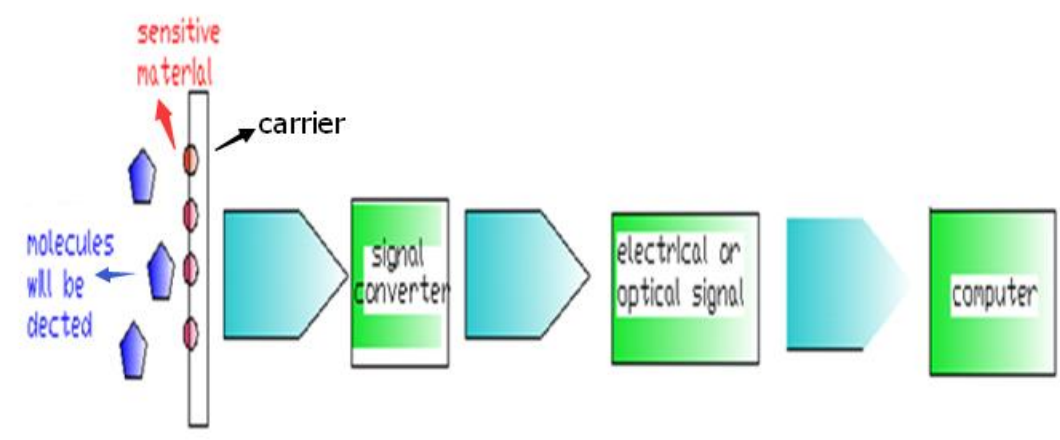

Figure 2. The principle of sensor for detecting material

\section{Applications of Electrospun Fibers in Sensors}

Applications in Biosensors. Biosensor which has important significance for biological sciences and medical diagnosis is a kind of analysis tools or systems contianing biological recognition element. Since Clark and Lyonsll [4] proposed to use membrane containing enzyme for the conversion of urine and glucose into products which can be detected by the $\mathrm{pH}$ electrode or the oxygen electrode in 1960s, researchers have done a lot of studies on the biological sensors. The technique of immobilizating biosensing materials is a key factor which directly affects the performances of the biosensors for detecting materials. Due to the electrostatic spinning fibers have the advantages of small structure size, large specific surface area and high porosity, using the nanofibers as biological sensitive materials carrier can greatly improve the sensitivity and response time of the biosensor. Applications of electrospun fibers in enzyme biosensors are very typical, meanwhile the applications of electrospun fibers immobilizating nonbiological substances (mainly metaloxide) for biosensors to detect biological molecular have appeared in large numbers. This section mainly introduces biosensors based on immobilizating enzyme, metaloxide and biological material on electrospun fibers respectively.

Applications in Gas Sensors. With the intensification of harmful gas pollution, the researches of gas sensors have become a hot topic .In recent decades, researchers have developed various types of gas sensors such as semiconductor type [5], solid electrolyte type, contact combustion type, optical type, surface acoustic wave type. In recent years, with the development of nanotechnique [6], the applications of nanomaterials in gas sensors are widely concerned. With nanostructure, high porosity, large specific surface area, electrospun fibers have been widely applied in gas sensors.

Applications in Metal Ion Sensors. At present, the means of detecting meatal ion have been various, such as ultraviolet spectroscopy, ion chromatography, atomic absorption spectrometry, electrochemical analysis and so on. With large specific surface area and high porosity, electrospun fibers have gradually become the preferred material for the detection of metal ion in recent years. 
Taking advantage of the good fluorescence of 1, 4-DH (1,4-dihydroxyanthraquinone ), Wang et [7] used electrospun membrane consisting of 1,4-DH and ethylic acid to detect $\mathrm{Cu}^{2+}$ and $\mathrm{Cr}^{3+}$ successfully. The specific experimental process and principle is shwon in Fig. 3. The fluorescence density of the 1, 4-DH/ ethylic acid membran decreased with increasing the concentration of $\mathrm{Cu}^{2+}$. On the contrary, the fluorescence density of the $1,4-\mathrm{DH} /$ ethylic acid/ $\mathrm{Cu}^{2+}$ membran increases with the raise of the concentration of $\mathrm{Cr}^{+}{ }^{+}$. This success of the metal ion sensor attributes to two key factors. One is the strong adsorption of nano electrospun membrane [8]. The other one is that the membrane plays a good role of a carrier for 1, 4-DH through electrospinning. This sensor based on fluorescence for detecting metal ion was the first one, which has great significance to the analysis and detection of metal ion.

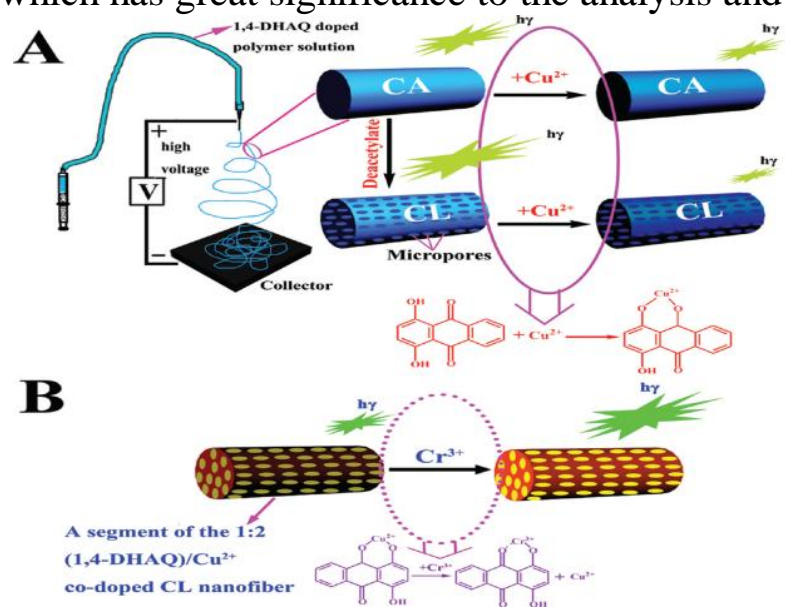

Figure 3. The specific experimental process and principle

Futhermore, Min et also obtained a metal ion sensor based on fluorescence and electrospun membrane. As a $\mathrm{Cu}^{2+}$ sensor, the electrospun membrane could reach a low limit detection of $1.1 \times 10^{-9}$ $\mathrm{M}$ and had a good selectivity. In addition, a kind of $\mathrm{Cu}^{2+}$ sensor with excellent repeatability had been obtained by Sun [9]. The PS electrospun membrane was deposited on the electrode, then a layer of gold film was sputtered onto the surface of electrospun membrane by a kind of vacuum sputtering technology. Finally, MPA (mercaptopropionic acid) was immobilized on the electrode by self ssembly technology. The test indicated that the limit detection could reach $5 \mathrm{ppm}$. What's more, the sensors could be repeatedly used for ten times.

Presently, a large number of electrospun fiber applications in the separation and collection of metal ion emerge, but there are not very many applications of electrospun fibers in metal ion sensor. With the improvement of electrostatic spinning technology, applications of electrospun fibers in metal ion sensors will be futher exploited.

\section{Conclusion and Prospects}

Due to high porosity and large surface area [10], electrospun fibers are widely applied in sensors. The sensors based on electrospun fibers always have higher sensitivity, faster response and lower limit detection than the sensors based on traditional film. In the futhure, two aspects should be enchanced to promote the applications of electrospun fibers in sensors. First, more attentions should be paid to research on the spinnability of the key sensing materials. Many sensing materials cannot be spinned by electrospinning or are prepared for electrospun nanofibers with poor morphology. Second, the efficiency of producing electrospun fibers should be improved. Due to good performance of the sensors based on electrospun fibers, this type of sensors will be large-scale applied in practical production in the futhure, but the efficiency based on the current electrospinning technique is far from large-scale production. With the delevlopment of electrospinning technique, it is believed that the sensors based on electrospun fibers will exhibit its more potentiality. 


\section{References}

[1] Park, Soo-Jin, et al. "Physical characteristics of titania nanofibers synthesized by sol-gel and electrospinning techniques." J. Eng. Fiber Fabr5.1 (2010): 50-56.

[2] Pramanik, Sumit, Belinda Pingguan-Murphy, and Noor Azuan Abu Osman. "Progress of key strategies in development of electrospun scaffolds: bone tissue." Science and Technology of Advanced Materials 13.4 (2012): 043002.

[3] Wu, Jingping, and Fan Yin. "Sensitive enzymatic glucose biosensor fabricated by electrospinning composite nanofibers and electrodepositing Prussian blue film." Journal of Electroanalytical Chemistry 694 (2013): 1-5.

[4] Marx, Sharon, et al. "Electrospun gold nanofiber electrodes for biosensors." Biosensors and Bioelectronics 26.6 (2011): 2981-2986.

[5] Liu, Guangyue, et al. "Improvement of sensitive $\mathrm{CuO}$ NFs-ITO nonenzymatic glucose sensor based on in situ electrospun fiber." Talanta 101 (2012): 24-31.

[6] Wu, J. P., and F. Yin. "Novel Hydrogen peroxide biosensor based on hemoglobin combined with electrospinning composite nanofibers." Analytical Letters 46.5 (2013): 818-830.

[7] Ahn, M-W., et al. "Gas sensing properties of defect-controlled ZnO-nanowire gas sensor." Applied Physics Letters 93.26 (2008): 263103.

[8] Zhang, Chunyan, et al. "Nanoporous polystyrene fibers functionalized by polyethylene mine for enhanced formaldehyde sensing." Sensors and Actuators B: Chemical 152.2 (2011): 316-323.

[9] Sun, Min, et al. "Self-assembled monolayer of 3-mercaptopropionic acid on electrospun polystyrene membranes for $\mathrm{Cu}<\sup >2+</$ sup $>$ detection." Sensors and Actuators B: Chemical 161.1 (2012): 322-328.

[10]Min, Minghua, et al. "Highly sensitive and selective $\mathrm{Cu}<\sup >2+</$ sup $>$ sensor based on electrospun rhodamine dye doped poly (ether sulfones) nanofibers." Sensors and Actuators B: Chemical 188 (2013): 360-366. 